\title{
Scheduling of a computer integrated manufacturing system: A simulation study
}

\author{
Nadia Bhuiyan ${ }^{1}$, Gerard Gouw ${ }^{1}$, Dayoosh Yazdi ${ }^{2}$ \\ ${ }^{1}$ Concordia University (CANADA), ${ }^{2}$ Poshkar (IRAN) \\ bhuiyan@alcor.concordia.ca; goum@encs.concordia.ca; dary_yazdi@,hotmail.com
}

Received February 2011

Accepted October 2011

\section{Abstract:}

Purpose: The purpose of this paper is to study the effect of selected scheduling dispatching rules on the performance of an actual CIM system using different performance measures and to compare the results with the literature.

Design/methodology/approach: To achieve this objective, a computer simulation model of the existing CIM system is developed to test the performance of different scheduling rules with respect to mean flow time, machine efficiency and total run time as performance measures.

Findings: Results suggest that the system performs much better considering the machine efficiency when the initial number of parts released is maximum and the buffer size is minimum. Furthermore, considering the average flow time, the system performs much better when the selected dispatching rule is either Earliest Due Date (EDD) or Shortest Process Time (SPT) with buffer size of five and the initial number of parts released of eight.

Research limitations/implications: In this research, some limitations are: a limited number of factors and levels were considered for the experiment set-up; however the flexibility of the model allows experimenting with additional factors and levels. In the simulation experiments of this research, three scheduling dispatching rules (First In/First Out (FIFO), EDD, SPT) were used. In future 
research, the effect of other dispatching rules on the system performance can be compared. Some assumptions can be relaxed in future work.

Practical implications: This research helps to identify the potential effect of a selected number of dispatching rules and two other factors, the number of buffers and initial number of parts released, on the performance of the existing CIM systems with different part types where the machines are the major resource constraints.

Originality/value: This research is among the few to study the effect of the dispatching rules on the performance of the CIM systems with use of terminating simulation analysis. This is also significant given the nature of the CIM systems that are mostly used to produce different parts in varying quantities and thus do not produce parts on a continuing basis. This research is amongst the first to study the combined effect of dispatching rule and the buffer size in the CIM systems where the job arrivals are predetermined and depend on the completion of the existing parts in the system. A description of how buffer size and initial part release is related to the performance of the CIM system under study for the studied priority dispatching rule is also provided.

Keywords: computer integrated manufacturing, flexible manufacturing systems, scheduling, dispatching rules, simulation

\section{Introduction}

In modern industrial settings, considering the tight competitive market, efficiency and superior performance are critical factors for companies to address. One approach that companies have taken in this regard is to increase the level of automation and computerization of their production system (Cagliano \& Spina, 2000; Nagalingam \& Lin, 1999; Theodorou \& Florou, 2008; Tseng, 2004). Flexible manufacturing systems (FMS) and computer integrated manufacturing (CIM) systems are examples of such approaches. A CIM system is defined as a system that focuses on the computer as the focal point of control of the factory, and covers the computerization of the fabrication and assembly processes, as well as information flow for production control, quality, maintenance, material handling, and inventory control in a completely integrated system (Asfahl, 1992). An FMS 
"takes advantage of the flexibility of the robots, NC machine tools, industrial logic controllers and microprocessors" to create an overall flexible system (Asfahl, 1992).

Flexible manufacturing systems are increasingly being used in manufacturing for their advantages of flexibility, quality, reduced labor and inventory cost in the era of continuous improvements and frequent turnaround initiatives. However the main disadvantage of the FMS is the high initial investment required. For this reason, it is of utmost importance for firms to ensure the economical justification to acquire these systems. The production scheduling problem is one of the main areas of research in the study of FMSs. The objective is to come up with the best production sequence for jobs by assigning the available resources within the existing constraints such that the desired performance of the system is maximized (Holthaus \& Rajendran, 1997). Scheduling performance is usually evaluated based on certain criteria that can be classified in two general areas: meeting the due date of the ordered products and increasing the productivity of the system.

In many situations, production scheduling is done through the use of dispatching rules due to their effectiveness and ease of use. Dispatching rules are predefined rules that prioritize the jobs waiting for processing. Many studies have shown that system performance varies to a great degree for different shop environments and thus no dispatching rule has been found to be optimal for all planning and scheduling problems (Montazeri \& Van Wassenhove, 1990). Furthermore, the performance of scheduling rules depends on the performance criteria under consideration and also the arrangement of the production system. The body of literature in this area is sometimes contradictory since the experimental settings and assumptions of these studies are not the same. Consequently, for each FMS there has to be a separate scheduling study to find the best dispatching rule to accommodate the desired measure of performance.

In this paper, we evaluate the performance of selected dispatching rules for different operations against different performance measures on an existing CIM facility using a simulation model. The remainder of this paper is organized as follows. Section 2 provides a survey of the scheduling literature, their classification and the method used to address them with the main focus in the area of the priority dispatching rules. Section 3 provides an overview of the system used in the study, including its layout, control system, communication network and programming. The next section covers the design of experiments to evaluate the performance of the rules by using both a simulation model and the real system. Section 5 presents and discusses the results of the experiments for the simulation 
model and the real system, and finally, the last section presents concluding remarks, the highlights of the research and recommendations for future research.

\section{Production scheduling}

The production scheduling problem is one of the main areas of research on FMSs. In general terms, the problem can be described as follows: given a set of required tasks, what is the best way to assign the available resources to the tasks, within the existing constraints that would maximize the desired performance of the system. Thus, a mechanism is required to make a decision on the priority of the tasks and create the sequence of the activities in the FMS. These activities normally include the selection of the next part to be processed by the machines when the machine becomes free, the selection of the next part to be released into the system, and the selection of the next part to be loaded on a transportation system. Scheduling problems can be classified based on different dimensions. Jones and Rabelo (1998) categorized them according to manufacturing environment, process complexity, scheduling criteria, parameters variability, and scheduling environment.

Dispatching rules have been used in the last few decades to address scheduling problems for their simplicity and ease of use (Drake et al., 1995; Mohanasunduram et al., 2003; Sabuncuoglu \& Lejmi, 1999). Dispatching rules can be classified into different categories based on different factors. To study the effect of the dispatching rules on the FMS, normally the measures of performance of the system are monitored. Examples of performance measures include average lateness, flow time, production cost, production consistency, job quality, job priority, machine and tool utilization, average work-in-process and average waiting time per part. However, the most used performance measures in the scheduling studies are lateness, flow time and tardiness (Blackstone et al., 1982).

In general, the main interest has been improving a shop's productivity while meeting the due dates. However no rule has been found to perform well and improve all performance measures. Thus the choice of dispatching rule depends on the performance measure as well as the characteristics of the system under study.

\section{Existing work}

The body of literature in this area is sometimes contradictory since the experimental settings and assumptions of these studies are often not the same. Consequently, for each FMS there has to be a separate scheduling study to find the best dispatching rule to accommodate the desired measure of performance. In 
general, there are two main performance objective categories that dispatching rules should improve, namely to increase productivity and to meet the due date of the job orders. When dealing with meeting job due dates, the performance measure which is usually used in the literature is mean job tardiness. In the following, the literature directly related to these two aspects of production shops will be reviewed.

Conway (1965a) considered a shop with nine machine groups each with a single machine. In this experiment, he reported results for over 30 dispatching rules. He used four different performance measures in studying the effect of dispatching rules. In total, Conway tested 16 different priority dispatching rules and concluded that the shortest processing time (SPT) rule performs relatively better than all other rules in general with respect to average job lateness and in-process-inventory for the four methods of due date assignment as described earlier. The SPT rule reduces mean flow time as follows: by giving priority to the jobs with short process times, it accelerates the progress of production of jobs at the expense of some jobs with long processing time. This way, in total the average flow time is reduced, but jobs with long processing time face long waiting times.

Montazeri and Van Wassenhove (1990) have also studied the effectiveness of the scheduling rules for various system performance measures using a discrete event simulator. They concluded that the SPT priority rule was the second best priority rule for the system under study in terms of average waiting time. No single scheduling rule was found to improve both average and variance of a job's waiting times. They also concluded that SPT based rules minimize average waiting times and LPT (Longest Process Time) based rules maximize machine utilization. Finally, no single scheduling rule was found to be the winner on all performance measures. They suggested that it is up to the user to choose the scheduling rules based on the performance measure that needs to be improved.

Choi and Malstrom (1988) described the use of a physical simulator as opposed to computer simulation as an analysis tool in the evaluation of scheduling dispatching rules in an FMS. They studied the performance of seven dispatching rules including Random, first in first out (FIFO) and SPT based on six performance measures including actual system effectiveness, total traveling time of parts, actual production output, total manufacturing throughput time, work-in-process inventory and total production lateness. They concluded that the Random rules had high values of actual system effectiveness and low values of production lateness, whereas the SPT had high values of the actual production output, low throughput 
time and low work-in-process inventory. However no rule was found to be best for all performance measures.

The greater part of the literature on scheduling problems considers the setup as insignificant or at most, part of the job's processing time. Although this assumption makes the analysis of the problem easier, in some cases where the setup time is significant, the generated solutions would not be the optimal especially when the setup time is not equal for all jobs. System setup includes activities such as adjusting tools, positioning process material in storage, tooling cleanup, setting the required jigs and fixtures and inspecting materials.

Based on a detailed literature review conducted in Yazdi (2006), the overall best simple dispatching rules among all other simple rules in order of their performance are SPT, EDD and FIFO. In this paper, we study the effect of these scheduling dispatching rules on the performance of an actual CIM system using different performance measures.

\section{Methodology}

The performance of different dispatching rules is studied through a simulation model of an existing CIM system based on the control logic that describes the operation of the system. ARENA simulator software is used for modeling the CIM system. Design of Experiments (D.O.E.) is used to study environment factors that influence the performance of the selected dispatching rules on the existing CIM system. The performance of the dispatching rules is evaluated and analyzed using ANOVA (analysis of the variance). Finally, the results from the study are compared with the results from the existing literature.

\section{System overview}

Typically, an FMS is able to produce a range of different products where the traditional product assembly lines normally produce a high volume of a limited number of products. FMSs are normally automated, where a central control system controls a number of CNC machines connected by a material handling system, usually automated guided vehicles (AGVs), or a conveyor system. The existing FMS under study consists of four workstations around a closed conveyor loop for part transportation among workstations as shown in Figure 1. 


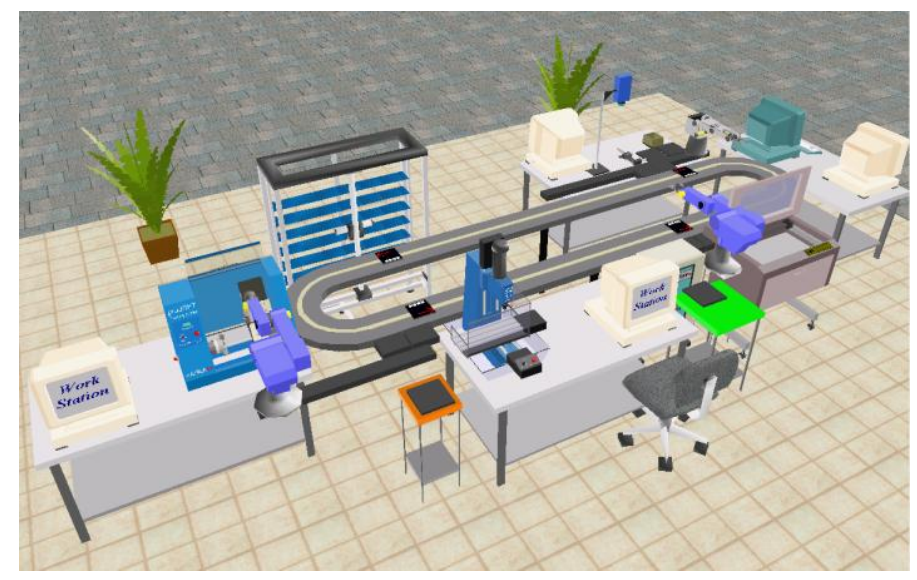

Figure 1. Layout of FMS

The workstations include an Automated Storage and Retrieval System Station (AS/RS), which is an automatic warehouse which supplies raw materials to the system, stores parts in intermediate stages of production, and holds finished products with its Cartesian robot; three machining stations where materials are shaped, a CNC station with a mill, a lathe, and a laser engraver station; an assembly and quality control (QC) station for inspection of parts using machine vision. Each machining station and the QC station have a serving robot and a buffer area to hold all jobs to be processed. The CNC station's buffer has the capacity of four parts and the laser engraver and QC stations' buffers each have the capacity of two parts. Each part type has a unique template to mount the part. Also, the conveyor system has six pallets where the templates (with mounted parts) are being placed on for transportation among stations. The shop floor control system (SFCS) monitors the location of the parts either separately, when they are being processed at a station, or in conjunction with a template when they are on pallets, in ASRS or in a buffer location. Details of the model can be found in Yazdi (2006).

\section{Design of experiments}

Design of Experiments (D.O.E.) is a structured approach in experimentation used to identify the significance of selected variables (factors) on the performance of the system under study. In this approach the required set of experimental runs is created based on the combinations of factors and the levels of these factors. The experiments are performed by measuring the performance measures of the system under study, while changing the values of other factors in the system. The point is to evaluate the impact of these changes.

In order to study the characteristics of the FMS and its production performance, it is necessary to find the factors which affect the system's performance. In order to 
avoid the significant increase in the number of simulation runs, the experiment must be designed carefully. In general, these factors can be classified into two categories: controllable or design factors, and uncontrollable factors. Some examples of uncontrollable factors include the number of machines, part mix, number of buffers etc.

The main design factors of interest in this study in the existing CIM system are defined dispatching rules. These rules are mainly used by the handling robots in the system to perform material-handling tasks according to the production plan. They are FIFO, Earliest Due Date (EDD), and SPT. These rules are well known sequencing algorithms which have been shown to be effective in specific production systems settings and/or used as a reference.

Two other factors are also considered in this study. The first factor is the system load (initial number of released parts), which is treated as an experimental factor to study the FMS for different levels of system congestion. The release time of each additional part from the AS/RS is not set in terms of time intervals, but rather in terms of the work (part) progress. An additional part is released to the system only when the previous part exits the system or reaches a specified stage in its production process. Thus the increase or decrease in the system utilization is set by the number of initial parts released into the system. The second factor is the number of buffers in the system. These factors are included in the experiment because they introduce variability in the CIM system. The introduction of variability into the system is important since the effectiveness of the dispatching rule should be proven under different conditions. Furthermore, these factors have also been used in similar studies that enable a comparison of the findings of the experiments with the literature in this field.

Three levels of the two factors, presented are suggested in Table 1 . These levels will allow the number of the total required simulation runs to remain in a feasible execution level and thus to be able to employ full factorial set-up of experiments.

\begin{tabular}{|c|r|rr|}
\hline \multirow{2}{*}{ Levels } & \multicolumn{3}{|l|}{ Factors for the experimental set-up } \\
\cline { 2 - 4 } & $\begin{array}{l}\text { Number of initially } \\
\text { released parts }\end{array}$ & $\begin{array}{l}\text { Number of buffers } \\
\text { in the system }\end{array}$ \\
\hline 1 & 3 & 3 \\
\hline 2 & 8 & 4 \\
\hline 3 & 12 & 5 \\
\hline
\end{tabular}

Table 1. Factor-level of The Experiment 
The first level of the Number of initially released parts factor is set to three since it is required to have a lineup of jobs needed to be processed in the system to be able to see the effect of the dispatching rules. Furthermore, upon constructing the simulation model it was discovered that a deadlock may occur in the system depending on the number of buffers in the system and the number of initially released parts. It was discovered that the maximum number of parts released should not exceed the number of available buffers plus nine. Under these circumstances, the minimum number of buffers is set to three and consequently the maximum number of released parts to twelve. The latter number also helps to keep each real production run in a manageable time frame. The second level of parts released is chosen to be a number between the first and last level (8).

The number of buffers in the system factor also has three levels. The first level is chosen to be three, as discussed above. Furthermore, since the main workstation under study in the existing system is the CNC and since this workstation has four buffers, the second level of buffers is set to four. The maximum level of this factor is set at five. Since the study uses two factors with three levels of severity along with 3 different scheduling dispatching rules, a $3 \times 3 \times 3=27$ full factorial D.O.E. set-up is formulated to evaluate the performance of the system under study. The combination of factor levels can be provided by any of the available commercial statistical software packages since it is essential to run a complete randomized experimental design. Randomization is important in any experimental design when it is uncertain that every major influence on the system has been included in the experiment. Even when all major influences have been identified and included in the experiment, unplanned complications can bias the results of an experiment. Thus, when comparisons are made among levels of a factor, randomization will tend to cancel the bias effect and the true factor effect will remain (Montgomery, 2001). The set-up of the runs is presented in Table 2.

To analyze the significance of the output of the D.O.E., analysis of variance (ANOVA) is conducted. In order to be able to perform an ANOVA analysis, at least two replications of each experiment are required, however to obtain more reliable values of the performance metrics, more replications are required. Kelton and Law (1982) and Schmeiser (1982) recommend ten replications, which was used in this study as well. Thus, for each dispatching rule and all the factor-level combinations, a single simulation run is replicated 10 times in the simulation model. Hence the number of total experiment treatments will amount to 270 ( 27 conditions $* 10$ experiment replications). 


\begin{tabular}{|c|c|c|c|}
\hline RUN No. & $\begin{array}{l}\text { Dispatching } \\
\text { Rule }\end{array}$ & $\begin{array}{l}\text { Number of } \\
\text { initially released } \\
\text { parts }\end{array}$ & $\begin{array}{l}\text { Number of } \\
\text { buffers in the } \\
\text { system }\end{array}$ \\
\hline 1 & FIFO & 3 & 3 \\
\hline 2 & FIFO & 3 & 4 \\
\hline 3 & FIFO & 3 & 5 \\
\hline 4 & FIFO & 8 & 3 \\
\hline 5 & FIFO & 8 & 4 \\
\hline 6 & FIFO & 8 & 5 \\
\hline 7 & FIFO & 12 & 3 \\
\hline 8 & FIFO & 12 & 4 \\
\hline 9 & FIFO & 12 & 5 \\
\hline 10 & EDD & 3 & 3 \\
\hline 11 & EDD & 3 & 4 \\
\hline 12 & EDD & 3 & 5 \\
\hline 13 & EDD & 8 & 3 \\
\hline 14 & EDD & 8 & 4 \\
\hline 15 & EDD & 8 & 5 \\
\hline 16 & EDD & 12 & 3 \\
\hline 17 & EDD & 12 & 4 \\
\hline 18 & EDD & 12 & 5 \\
\hline 19 & SPT & 3 & 3 \\
\hline 20 & SPT & 3 & 4 \\
\hline 21 & SPT & 3 & 5 \\
\hline 22 & SPT & 8 & 3 \\
\hline 23 & SPT & 8 & 4 \\
\hline 24 & SPT & 8 & 5 \\
\hline 25 & SPT & 12 & 3 \\
\hline 26 & SPT & 12 & 4 \\
\hline 27 & SPT & 12 & 5 \\
\hline
\end{tabular}

Table 2. Set-up of the Experimental Design Runs

\subsection{Production batch size}

The number of produced parts for each production cycle run is 12 jobs. Each production batch contains different part types. In this study, each production run consisted of three different part types. In order to be able to use the EDD based dispatching rule, it is necessary to have different parts with different due dates in each production cycle so that when the EDD dispatching rule is selected as the priority rule, it gives the priority to a part having the earliest due date.

\section{Characteristics of the system}

In this study, the existing FMS is regarded as a flow shop. However, several features which differentiate this system from the "standard" flow shop, as studied by Conway (1965a; 1965b), Rajendran and Holthaus (1999) and other researchers (Montazeri \& Van Wassenhove, 1990; Persi et al., 1999; Choi \& Malstrom, 1988; Hong \& Chou, 2002) are:

- Job inter-arrival time: The arrival of new parts to the system (release of additional part from the AS/RS) is set in terms of the progress of jobs in the system and not based on some form of time distribution. An additional part 
is released when the previous part exits the system or reaches a certain stage in the production process. This method is unlike the other research studies where the arrival of a new part is based on a certain time interval and usually treated as a system input parameter.

- System work load: By defining the Initial Quantity of the released parts to the system in the manufacturing order form in the OpenCIM control software, the number of parts to be released from the AS/RS when production begins is defined. This is the only variable that can be used to manipulate the system work load. Thus the increase or decrease in the system utilization is set by the number of initial parts released into the system. It should be noted that as the simulation runs showed, the maximum number of parts that can be released to the system without creating a deadlock for the system is less than 19. Thus this variable is set between the minimum of 3 (in order to have a queue of parts waiting to be processed in the system) and 18 . Consequently there is no possibility to set the work load of the system to a predetermined percentage like other research studies.

\subsection{Assumptions}

The following assumptions for the setup of the experiments for the existing CIM system are considered:

- All the operations will be performed based on the designed G-Code program where operation times at the CNC stations are fixed.

- The time required for tool changes is considered negligible with the help of an automatic tool changer (ATC).

- Due dates for each part type in each order are known.

- All the raw materials are available at the beginning of each production cycle and thus there are no inter-arrival times for raw materials.

- The raw material is the same for every product and comes in as cylinder type plastics.

- Priority ordering of parts is equal and is set to one (the highest priority). 
- Once an operation has begun, it should be completed before starting the next operation (the processing of only one operation on a given machine at a given instant is allowed).

- The resource requirements are predetermined and there are no alternatives.

- No machine breakdowns or tool failures are considered.

- The study does not include preventing blocking situations. However this effect may occur in the course of the experiment.

\section{Experimental conditions}

\subsection{Parts to be produced}

Three part types are produced in this system and their processing sequence is such that each job has two operations and each operation is done by a different machine. The processing time for each operation is assumed to be between two to ten minutes. Each of the part types follows a similar processing route and each production batch consists of equal numbers of each part.

\subsection{Due date setting}

Due dates can be set either externally or internally. When due dates are set externally, the scheduling system function is to arrange and prioritize the production plan to accommodate the predefined date. Internally set due dates, however, drive from the production load, manufacturing capacity and type of jobs being produced in the system. In the existing system the due dates are considered to be set externally and thus the production plan is arranged to meet the deadline. Furthermore, in order to be able to use the due date based scheduling dispatching rule (EDD), it is essential to have different due dates for different part types in each production run cycle. Therefore, Table 3 will be used as the production order reference for each production run. This due date arrangement will be the same for all experimental run settings.

\begin{tabular}{|c|r|r|r|}
\hline Order No. & Part Type & \multicolumn{1}{l|}{ Order Date } & Due Date Priority \\
\hline 1 & 70 & 1 & 3 \\
\hline 2 & 71 & 1 & 2 \\
\hline 3 & 72 & 1 & 4 \\
\hline
\end{tabular}

Table 3. Production Order for Production Runs 


\subsection{Transportation time}

It can be expected that the utilization of machines is relatively low due to the fact that the total expected transportation time is rather significant compared to the total expected processing time for parts. Table 4 shows the approximate transportation times between workstations in the existing FMS.

\begin{tabular}{|l|r|r|}
\hline \multirow{2}{*}{ Location } & \multicolumn{2}{|c|}{ Next Location } \\
\cline { 2 - 3 } & WS1*: AS/RS & WS2*: CNCs \\
\hline WS1: AS/RS & $0 \mathrm{~min}$ & $1 \mathrm{~min}$ \\
\hline WS2: CNCs & \multicolumn{2}{|c|}{$3 \mathrm{~min}$} \\
\hline \multicolumn{2}{|c|}{$*$ Work station $1 * *$ Work station 2} \\
\hline
\end{tabular}

Table 4. Transportation time between work stations

\subsection{The performance measures}

The effectiveness of the dispatching rules can be compared using the system on the basis of performance criteria. In general, the following performance criteria have been observed in the existing literature in this field: criteria based on due date, criteria based on flow time, criteria based on in-process parts. The performance measures used in the current experiment are as follows (OpenCIM, 2004):

- Total Run Time: the time period of the manufacturing cycle.

- Machine Efficiency: the efficiency of each machine in the system. It is defined as the total process time divided by the total manufacturing time of the machine.

- Average Flow Time: the average time that it takes to manufacture a product.

\section{Simulation model}

This study simulates the existing FMS where we have a limited production capacity, therefore the terminating simulation method is used.

\subsection{Model overview}

Raw materials are stored in the AS/RS station. Upon start of the production cycle, according to the initial number of parts released number setup, raw material parts are taken from the AS/RS and put on the conveyor's pallet by way of the AS/RS serving robot. The conveyor then delivers the raw material to workstation 1 . The parts are now taken from the conveyor and put on the workstation 1 buffer by the 
robot serving that station. The raw material is then selected from the queue, based on the selected dispatching rule to be processed at the CNC Lathe. Upon completion of this operation, the partially finished parts are moved to the CNC Mill for the final machining operation. The robot now puts the finished product back on the workstation 1 buffer. Finally the robot places the finished product on a pallet and the conveyor delivers it back to the AS/RS station for final storage. A diagrammatic representation of these tasks is presented in Figure 2. A detailed description of the components is given in the following section.

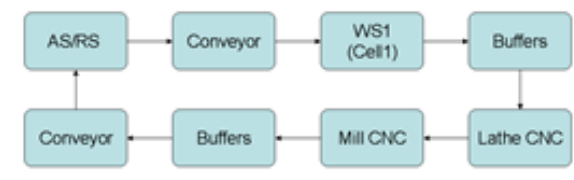

Figure 2. Part flow in the system

The existing FMS under study consists of four workstations around a closed conveyor loop for part transportation among workstations. The existing work stations are as follows:

- An AS/RS Station (Automated Storage and Retrieval System) supplies raw materials to the system, stores parts in intermediate stages of production, and holds finished products using its robot.

- A Machining Station, where materials are shaped. There are two CNC machines (a Mill and a Lathe) in the system.

- A Laser Engraver Station

- An Assembly and Quality Control (QC) Station for assembly and inspection of parts using machine vision

Note that the laser engraver and the Assembly and QC station are not part of the simulation study. Furthermore, each machine station and the QC station have a serving robot and a buffer area to hold jobs that are waiting to be processed. Once a part is released to the system by the AS/RS based on the processes it visits different machines and equipment in the system. Scheduling rules prioritize these jobs on a machine. It is possible to assign different priority dispatching rules to each machine in the system, however since the interest of this research is to evaluate the performance of each dispatching rule separately, for each simulation run same dispatching rule is assigned to all the equipment in the system. 


\subsection{Model verification and validation}

Once a working model is created, it should be verified and then validated. Verification is to ensure that the model behaves as intended. Verification essentially is debugging the model in such a way that it would run to completion without having logical or syntax errors. Validation on the other hand is to ensure that the model behaves the same as the real system which is quite different in nature. The most common sources of problem in this respect are wrong assumptions, wrong input data, over-simplification of the system and limitation of the software. Initially, the verification of the model was conducted by way of using the "Highlight Active Module" animation option in the Arena software. In this regard the following observations were made to ensure the correct behavior of the model:

- the number of entities entering and exiting the system,

- the queues and the number of entities in them, especially the buffer queue,

- the machines' status, and

- the simulation run time

For validation of the model, the real FMS was setup to run with one part type, an initial part release of three, four buffers and SPT as selected dispatching rule with five replications. Next, the model was run under the same conditions. Table 5 shows the comparison of the total run time and machine efficiency of the real system and simulation runs. As can be seen the simulation model results agree with the real system runs.

\begin{tabular}{|c|r|r|r|r|}
\hline \multirow{2}{*}{ Run \# } & \multicolumn{2}{|c|}{ Total Run Time (Min) } & \multicolumn{2}{c|}{ Machine Efficiency (\%) } \\
\cline { 2 - 5 } & Real & Simulation & Real & Simulation \\
\hline 1 & 171 & 174 & 50.76 & 50.03 \\
\hline 2 & 179 & 172 & 49.17 & 50.05 \\
\hline 3 & 171 & 173 & 49.89 & 49.46 \\
\hline 4 & 168 & 177 & 50.17 & 49.49 \\
\hline 5 & 175 & 169 & 50.01 & 49.46 \\
\hline Average & $\mathbf{1 7 3}$ & $\mathbf{1 7 3}$ & $\mathbf{5 0 . 0 0}$ & $\mathbf{4 9 . 7 0}$ \\
\hline
\end{tabular}

Table 5. Comparative results from real system and simulation runs

\section{Model results}

Dispatching rule, number of initial parts released and number of buffers are variables in the main study. As explained earlier, a total of 270 runs are made. Table 6 shows the first 15 simulation runs. Column one marks the related order number in the standard 270 factor-level setup where these factor-level setup runs 
are randomized by the D.O.E. software. For example, the first simulation run made (column 2) is standard 251 (column 1), which has a setup of EDD as the dispatching rule, 12 as the number of initially released parts and 5 buffers. The simulation model calculates the total run time, maximum queue length, production cost, machine efficiency and means flow time. The results of the simulation runs for total run time, machine efficiency and mean flow time are analyzed further in the following section.

\begin{tabular}{|c|c|c|c|c|c|c|c|c|c|}
\hline & & Factor 1 & Factor 2 & Factor 3 & $\begin{array}{c}\text { Response } \\
1\end{array}$ & \begin{tabular}{|c} 
Response \\
2
\end{tabular} & Response 3 & $\begin{array}{c}\text { Respons } \\
\text { e } 4\end{array}$ & $\begin{array}{c}\text { Response } \\
5\end{array}$ \\
\hline Std & Run & $\begin{array}{c}\text { A: } \\
\text { Dispatching } \\
\text { Rule }\end{array}$ & $\begin{array}{l}\text { B: Initial } \\
\text { Part } \\
\text { Release }\end{array}$ & C: Buffer & $\begin{array}{c}\text { Total Run } \\
\text { Time }\end{array}$ & $\begin{array}{c}\text { Maximum } \\
\text { Queue } \\
\text { Length }\end{array}$ & $\begin{array}{l}\text { Production } \\
\text { Cost }\end{array}$ & $\begin{array}{l}\text { Machine } \\
\text { Efficiency }\end{array}$ & $\begin{array}{c}\text { Mean } \\
\text { Flow Time }\end{array}$ \\
\hline & & name & Number & Number & Min & Number & $\$$ & $\%$ & $\min$ \\
\hline 251 & 1 & EDD & 12 & 5 & 161.20 & 5 & 80.60 & 51.05 & 91.530 \\
\hline 249 & 2 & FIFO & 12 & 5 & 159.88 & 5 & 79.94 & 51.06 & 94.499 \\
\hline 77 & 3 & EDD & 12 & 3 & 161.22 & 3 & 80.61 & 52.69 & 92.854 \\
\hline 176 & 4 & SPT & 12 & 4 & 161.38 & 4 & 80.69 & 50.55 & 93.091 \\
\hline 181 & 5 & FIFO & 3 & 5 & 162.13 & 2 & 81.07 & 50.92 & 94.813 \\
\hline 32 & 6 & FIFO & 8 & 3 & 158.43 & 3 & 79.21 & 51.68 & 94.719 \\
\hline 209 & 7 & SPT & 3 & 5 & 162.40 & 2 & 81.20 & 49.71 & 93.858 \\
\hline 240 & 8 & SPT & 8 & 5 & 160.56 & 5 & 80.28 & 50.91 & 91.698 \\
\hline 22 & 9 & SPT & 3 & 3 & 161.07 & 2 & 80.53 & 50.26 & 94.833 \\
\hline 179 & 10 & SPT & 12 & 4 & 161.64 & 4 & 80.82 & 50.35 & 91.941 \\
\hline 21 & 11 & SPT & 3 & 3 & 164.74 & 2 & 82.37 & 50.28 & 95.233 \\
\hline 35 & 12 & FIFO & 8 & 3 & 157.24 & 3 & 78.62 & 51.31 & 92.494 \\
\hline 242 & 13 & FIFO & 12 & 5 & 158.73 & 5 & 79.36 & 50.93 & 91.906 \\
\hline 151 & 14 & FIFO & 12 & 4 & 159.70 & 4 & 79.85 & 52.46 & 95.068 \\
\hline 264 & 15 & SPT & 12 & 5 & 163.74 & 5 & 81.87 & 50.63 & 93.420 \\
\hline
\end{tabular}

Table 6. Partial results from the main simulation model runs

\section{Statistical analysis method and analysis of results}

The simulation study used for the experiments in this study is a terminating system simulation, since the manufacturing system has low volume production capacity for each run cycle due to the limited storage capacity of the AS/RS. After completion of each production batch, the system should be stopped, the final product collected from the AS/RS and new raw materials placed for the next production cycle. The significance of the experimental design alternatives is interpreted by way of statistical analysis (Montgomery, 2001; Chung, 2004) which is explained in the following. In the following sections, the results of the conducted experiments on the performance of the selected scheduling dispatching rules on the simulation model of the existing CIM System are presented. Three performance measures, Total Run Time, Average Parts Flow Time and Average Machine Efficiency were used in the 
experiments. ANOVA on all the collected performance measures is conducted followed by the residual analysis for model validation. Finally, plots of factors' effects and interactions presented to analyze the significance of each factor and their interactions.

\subsection{ANOVA on average flow time}

Table 7 shows the result of ANOVA on Average Flow Time for all part types used in the experiment including the P-value of the ANOVA F-test.

\begin{tabular}{|l|r|r|r|r|r|r|}
\hline Source & $\begin{array}{l}\text { Sum of } \\
\text { Squares }\end{array}$ & df & $\begin{array}{l}\text { Mean } \\
\text { Square }\end{array}$ & $\begin{array}{l}\text { F } \\
\text { Value }\end{array}$ & $\begin{array}{l}\text { p-value } \\
\text { Prob > F }\end{array}$ & \\
\hline Model & 474.76 & 18 & 26.38 & 13.13 & $<0.0001$ & significant \\
\hline $\begin{array}{l}\text { A-Dispatching } \\
\text { Rule }\end{array}$ & 99.67 & 2 & 49.84 & 24.81 & $<0.0001$ & significant \\
\hline $\begin{array}{l}\text { B-Initial Part } \\
\text { Release }\end{array}$ & 294.20 & 2 & 147.10 & 73.23 & $<0.0001$ & significant \\
\hline C-Buffer & 18.55 & 2 & 9.28 & 4.62 & 0.0107 & significant \\
\hline AB & 47.21 & 4 & 11.80 & 5.88 & 0.0002 & significant \\
\hline AC & 0.61 & 4 & 0.15 & 0.075 & 0.9896 & \\
\hline BC & 14.53 & 4 & 3.63 & 1.81 & 0.1278 & \\
\hline Residual & 504.19 & 251 & 2.01 & & & not significant \\
\hline Lack of Fit & 0.98 & 8 & 0.12 & 0.059 & 0.9999 & \\
\hline Pure Error & 503.21 & 243 & 2.07 & & & \\
\hline Cor Total & 978.96 & 269 & & & &
\end{tabular}

Table 7. ANOVA for the average flow time

The model is significant at the $99 \%$ level ( $F$ value 13.13 , $p$-value $<0.00001)$. The above table also shows the p-value of the ANOVA F-test on Average Flow Time for all experimental factors and interactions. High $F$ values identify the significant factors and interactions. In this case $A, B, C$ and $A B$ are significant model terms. The $F$ value of 4.62 indicates that there is an interaction between dispatching rule and initial part release. Summary statistics for the model including the standard deviation associated with the experiment, overall average of all the response data (Mean), Coefficient of Variation (C.V.) and Predicted Residual Error Sum of Squares (PRESS) are presented below.

\begin{tabular}{|c|c|c|c|}
\hline & $\begin{array}{l}\text { Average } \\
\text { Flow Time }\end{array}$ & $\begin{array}{l}\text { Machine } \\
\text { Efficiency }\end{array}$ & $\begin{array}{l}\text { Total Run } \\
\text { Time }\end{array}$ \\
\hline Std. Dev & 1.42 & 0.63 & 2.03 \\
\hline Mean & 93.31 & 51.24 & 160.60 \\
\hline C.V. \% & 1.52 & 1.23 & 1.26 \\
\hline PRESS & 583.41 & 115.91 & 1191.29 \\
\hline
\end{tabular}

Table 8. Summary statistics on average flow time, machine efficiency and total run time

As was indicated previously, the main assumption in conducting an ANOVA analysis is that the errors are normally and independently distributed. To verify the ANOVA analysis and check the normality assumption, the plots of normal probability of 
residuals and residuals versus predicted are presented below. If the distribution of errors is normal, the normal plot of residuals should form a straight line, indicating no abnormalities. In this case, the normality assumption is satisfied as shown in Figure 3.

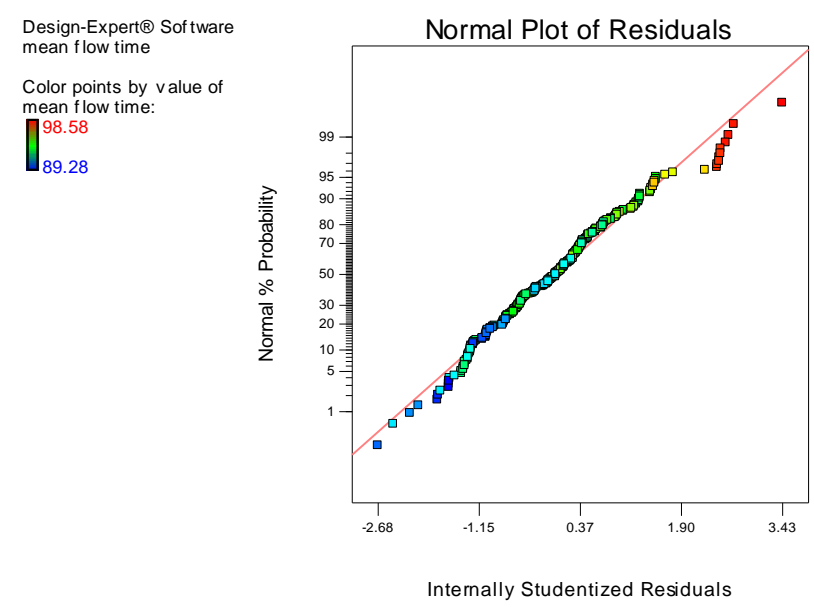

Figure 3. Normal plot of residuals

Figure 4 is a plot of the residuals versus the predicted response values. If the model is correct and if the constant variance assumption is satisfied, the residuals should have no patterns. The graph illustrates that there is no clear pattern and thus the constant variance assumption is satisfied. Having the model validated, concluding that the residual analyses do not reveal any problems, significant factor effects should be looked at. Figures 5, 6, and 7 show the average flow time, initial part release and dispatching rule for varying buffer sizes.

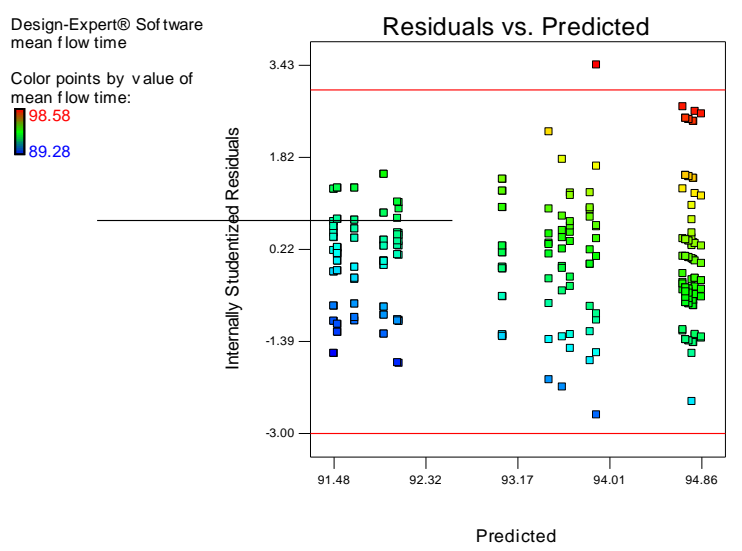

Figure 4. Plot of residuals versus the predicted response values

Figures 5 to 7 show the interaction between the initial part release factor and the selected dispatching rule. As can be seen, all the main factors have a sizeable effect 
on the mean flow time of the parts. When the initial part release is set at three (red line), the selected dispatching rule essentially has no effect on the mean flow time. This result can be justified by the fact that by having only three parts in the system, there will not be enough parts to form a substantial queue in such a way that the effect of different priority rules can be realized. However this is not the case for the other levels of the initial part release factor. In these cases the effect of the selected dispatching rule is identifiable. It can be seen that the EDD and SPT rules reduce the mean flow time of the parts almost the same way compared to the FIFO rule. The similarity of the EDD and SPT rules for the model can be explained by the fact that the simulator uses the lowest value of the designated attribute (Due Date and Process Time) to select the next part in the queue; hence these rules seem to behave similarly at the setting levels.

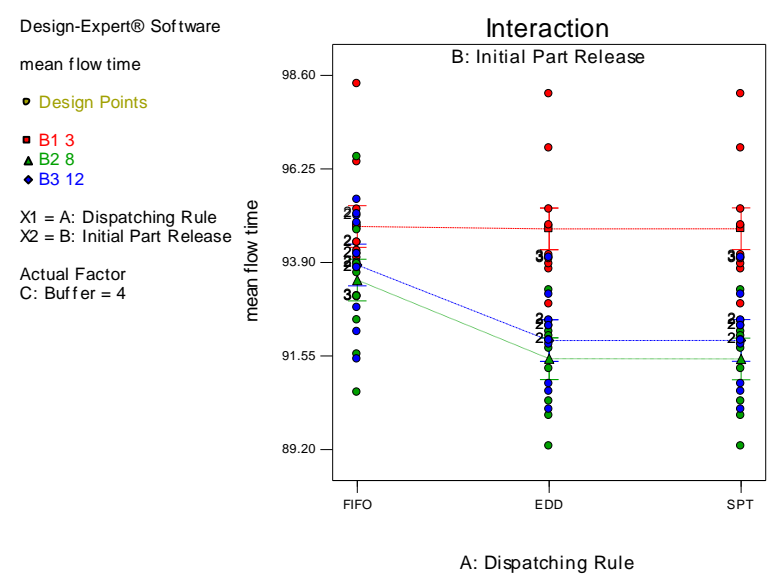

Figure 5. Average flow time, initial part release $\&$ dispatching rule

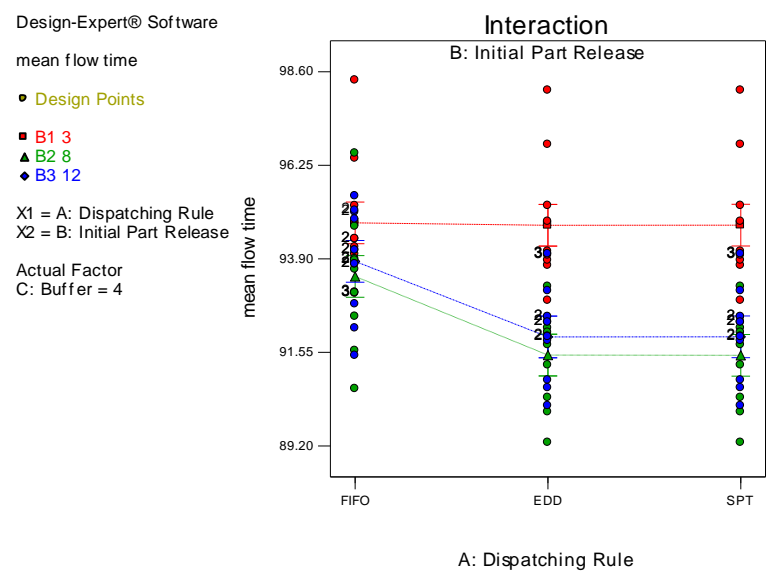

Figure 6. Average flow time, initial part release \& dispatching rule 


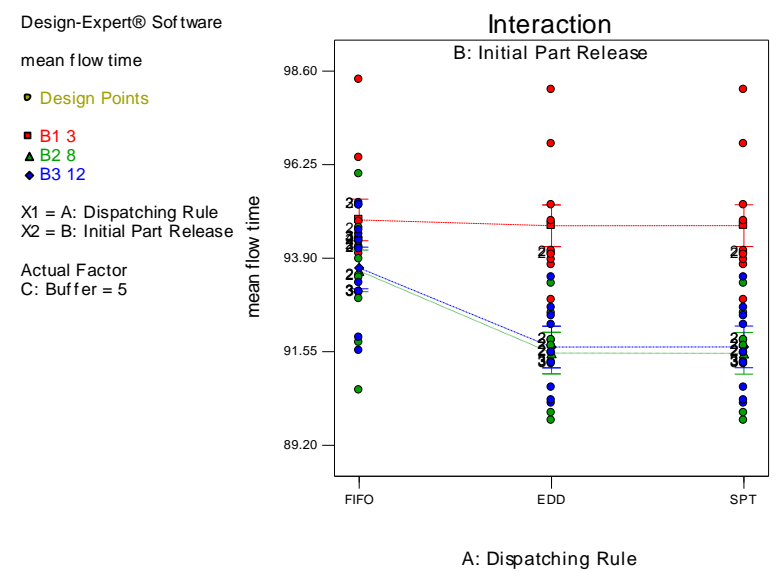

Figure 7. Average flow time, initial part release $\&$ dispatching rule

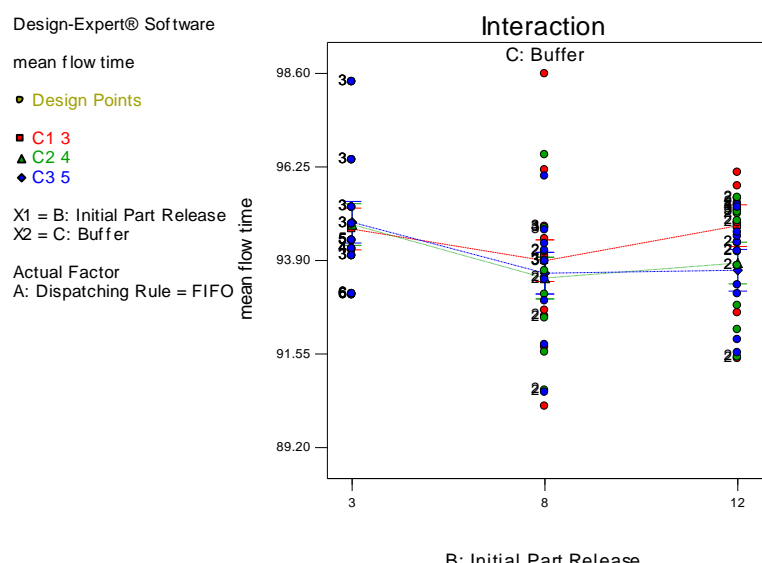

Figure 8. Average flow time, initial part release $\&$ buffer size for FIFO

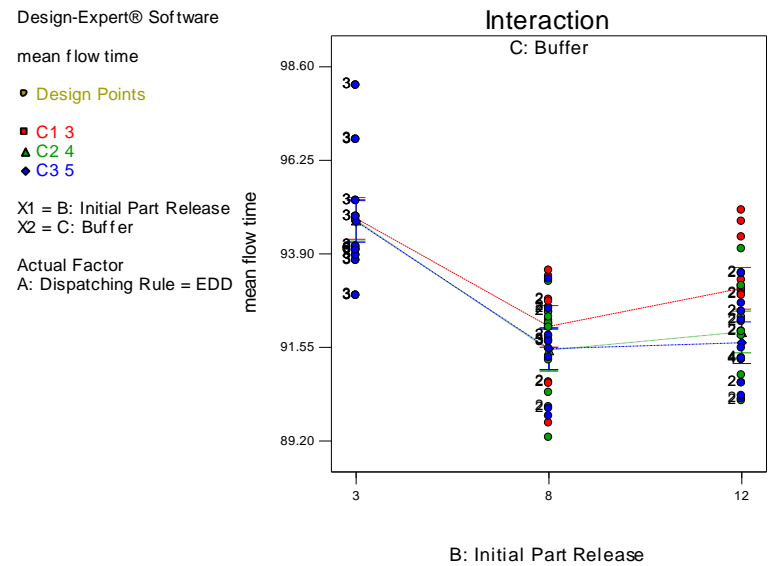

Figure 9. Average flow time, initial part release \& buffer size for EDD 


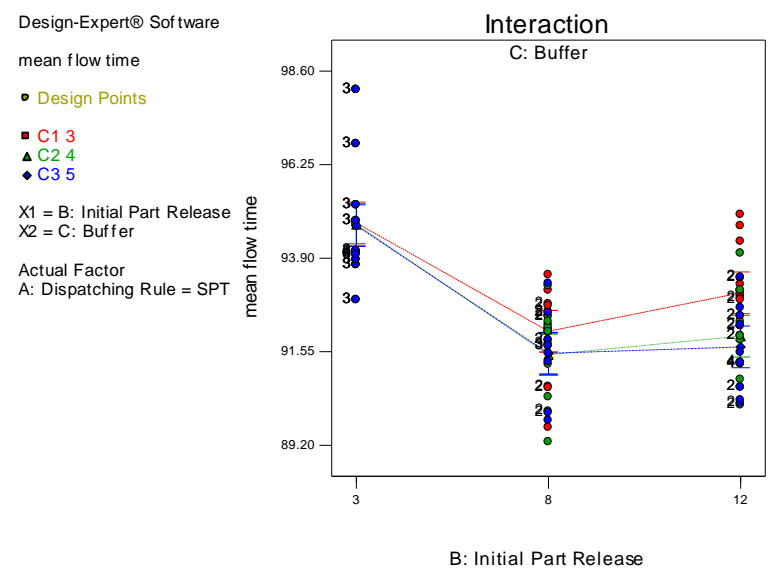

Figure 10. Average flow time, initial part release \& buffer size for SPT

Finally the effect of the number of the buffers in the system can also be seen in Figures 8 to 10 .

Comparing the graphs demonstrates that as the number of buffers increases in the system the effect of the dispatching rules becomes more pronounced at the higher level of initial part release (blue line). This effect is quite logical; by having a larger queue (bigger buffer size) the effect of the dispatching rule is more pronounced. Furthermore, from the above graphs it can be noticed that as the number of buffers in the system changes, the part mean flow time also change. At a given level of buffer size, there is an optimum number for initial part release which would minimize the part mean flow time. A lower level of initial part release would increase the mean flow time for inefficiency and extra capacity (extra buffers) and also the fact that after the initial part release, each new part enters the system only when a finished part exits the system. At the higher level of initial part release, part mean flow time increases due to the congestion in the system. In Figure 8 , the initial part release of 8 has the lowest mean flow time and thus it is the optimum number. As the number of buffers increases, although the mean flow time reduces but the reduction for the initial part release of 12 is more pronounced that is quite logical. The increase in the number of buffers shifts the optimum number if initial part release toward higher number of initial part release. Consequently the increase in the number of buffers, shifts the initial parts release of 8 (green line) down but not as much as the initial parts release of 12 (blue line).

From the above interaction plots, the following can be noted: 
- The performance gap between the buffer size of 3 on the one hand and a buffer size of 4 and 5 on the other hand becomes wider moving from the FIFO dispatching rule to the other two dispatching rules.

- Overall, moving from the FIFO dispatching rule to the other two dispatching rules, reduces the average flow time of parts.

- Initial part release has an interesting effect on the system performance. When going from an initial part release of 3 to 8 , the mean flow time of parts reduces markedly. However from that point the mean flow time starts to rise. This effect can be explained by the fact that at the level of 3 of initial part release, there is unused capacity in the CIM system which contributes to the longer mean flow time of the parts in the system. Also in the developed model, after the initial release of the parts, each new part enters the system once a final product leaves the system. However at a level of 8 of initial part release and on, there is no extra capacity in the system. Thus increasing the number of initial part release would create a longer queue and consequently increases the parts waiting time and thus average flow time of parts.

In summary, the system performs much better considering the Average Flow Time when the selected dispatching rule is either EDD or SPT with buffer size of five and initial part release of eight.

\subsection{ANOVA on machine efficiency}

\begin{tabular}{|l|r|r|r|r|r|r|}
\hline Source & $\begin{array}{l}\text { Sum of } \\
\text { Squares }\end{array}$ & \multicolumn{1}{c|}{ df } & \multicolumn{1}{l|}{$\begin{array}{l}\text { Mean } \\
\text { Square }\end{array}$} & $\begin{array}{l}\text { F } \\
\text { Value }\end{array}$ & $\begin{array}{l}\text { p-value } \\
\text { Prob > F }\end{array}$ & \\
\hline Model & 114.48 & 18 & 6.36 & 15.94 & $<0.0001$ & significant \\
\hline $\begin{array}{l}\text { A-Dispatching Rule } \\
\text { B-Initial Part } \\
\text { Release }\end{array}$ & 4.75 & 2 & 2.38 & 5.96 & 0.0030 & \\
\hline C-Buffer & 74.75 & 2 & 37.38 & 93.66 & $<0.0001$ & significant \\
\hline $\boldsymbol{A B}$ & 19.00 & 2 & 9.50 & 23.81 & $<0.0001$ & significant \\
\hline AC & 0.89 & 4 & 0.22 & 0.56 & 0.6927 & \\
\hline BC & 0.068 & 4 & 0.017 & 0.042 & 0.9966 & \\
\hline Residual & 15.01 & 4 & 3.75 & 9.40 & $<0.0001$ & significant \\
\hline Lack of Fit & 100.17 & 251 & 0.40 & & & not significant \\
\hline Pure Error & 0.52 & 8 & 0.065 & 0.16 & 0.9958 & \\
\hline Cor Total & 99.65 & 243 & 0.41 & & & \\
\hline
\end{tabular}

Table 9. ANOVA Analysis for machine efficiency

Table 9 shows the results of the ANOVA analysis on Machine Efficiency including the $\mathrm{p}$-value of the ANOVA F-test. With an $\mathrm{F}$ value of 15.94 , the model is significant at the $99 \%$ level ( $p$-value<0.0001). The table also shows the $p$-value of the ANOVA Ftest on Machine Efficiency for all experimental factors and interactions. High $F$ 
values identify the significant factors and interactions. In this case B, C and BC are significant model terms. The $F$ value of 9.4 indicates that there is an interaction effect between buffer size and initial part release.

A summary of the statistics for the model is presented in Table 8.

To verify the ANOVA analysis, the plots of normal probability of residuals and residuals versus predicted values are presented in Figures 11 and 12 . No abnormalities can be seen in the following normal plot, thus the normality assumption is satisfied.

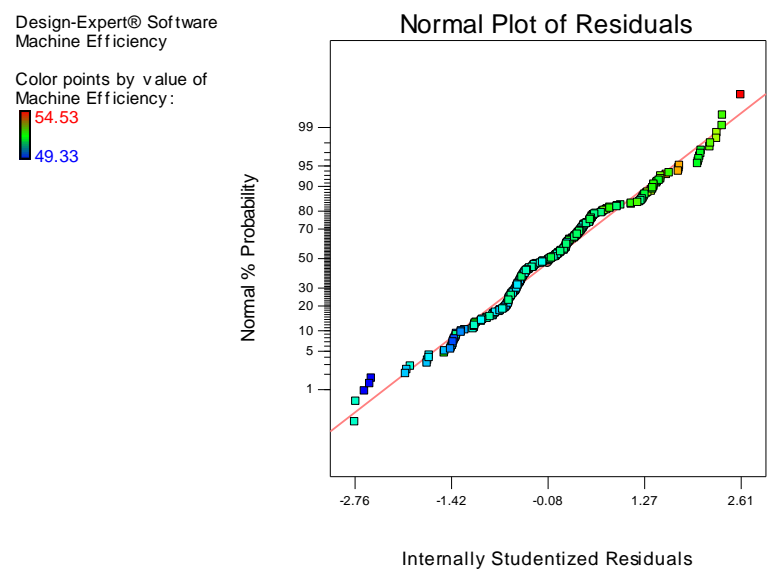

Figure 11. Normal plot of residuals for the ANOVA test on machine efficiency

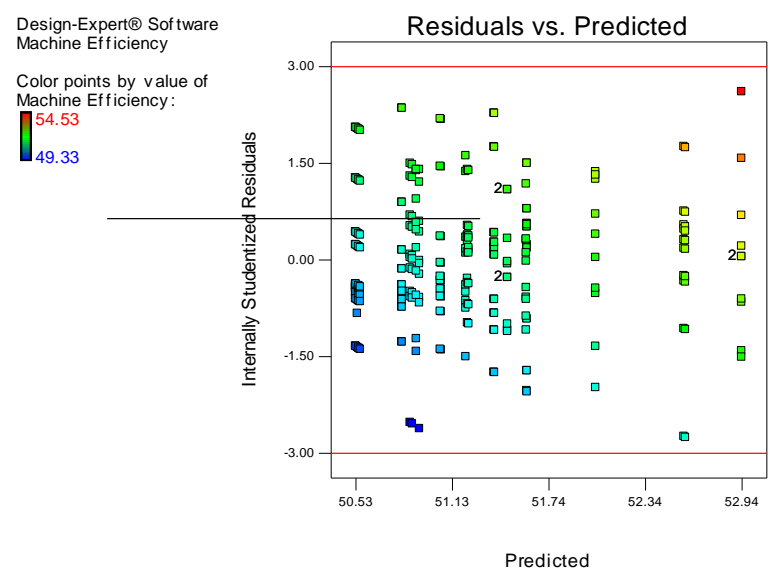

Figure 12. Residuals versus predicted values for machine efficiency 


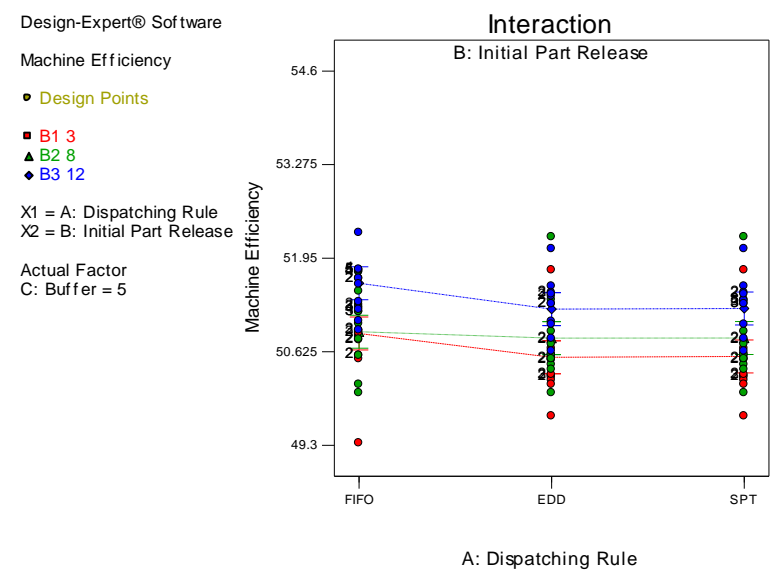

Figure 13. Machine efficiency initial part release $\&$ dispatching rule

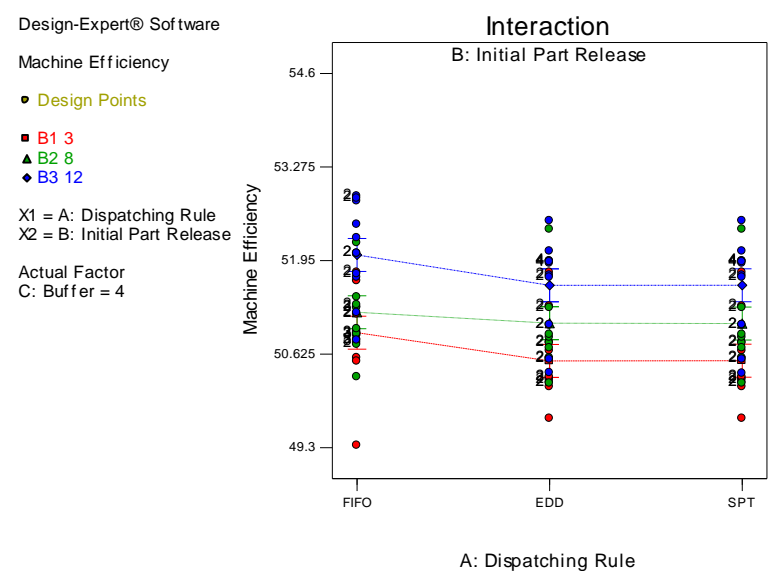

Figure 14. Machine efficiency initial part release $\&$ dispatching rule

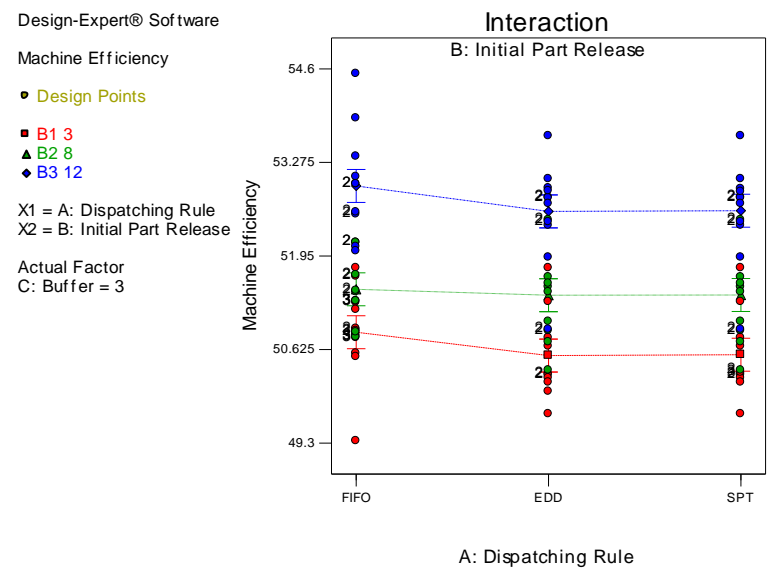

Figure 15. Machine efficiency initial part release \& dispatching rule

Figure 13 to 15 show the interaction between the initial part release factor and the selected dispatching rule. It can be seen the selected dispatching rule does not 
have a sizeable effect on the machine efficiency. However this is not the case for the other two factors. It can be seen that a lower number of initial part release reduces the machine efficiency. This can be justified by the fact that as the number of initial part release increases, the system workload increases which results in more use of the machines and thus a higher machine efficiency.

Finally the effect of the number of buffers in the system can also be seen in these graphs. Comparing the graphs shows that the as the number of buffers increases in the system the effect of the initial release becomes less (the distance between graphs reduces). This effect is quite logical; by having a smaller buffer size, the machines are used more efficiently to finish the job order. This effect is more pronounced when the initial part release is the highest and the buffer size the lowest (blue line in Figure 15). The above interaction plots once again verify the conclusion that is already made. It can be noticed that the selected dispatching rule does not have a marked effect while the effect of the increase in buffer size becomes more pronounced when the number of initial part release increases.

In summary, the system performs much better in terms of the Machine Efficiency when the initial part release is maximum and the buffer size is minimum.

\subsection{ANOVA on total run time}

Table 10 shows the result of Analysis of Variance analysis on the Total Run Time including the $p$-value of the ANOVA F-test.

\begin{tabular}{|c|c|c|c|c|c|c|}
\hline Source & $\begin{array}{l}\text { Sum of } \\
\text { Squares }\end{array}$ & df & $\begin{array}{l}\text { Mean } \\
\text { Square }\end{array}$ & $\begin{array}{l}\text { F } \\
\text { Value }\end{array}$ & $\begin{array}{l}\text { p-value } \\
\text { Prob > F }\end{array}$ & \\
\hline Model & 164.54 & 18 & 9.14 & 2.23 & 0.0035 & significant \\
\hline A-Dispatching Rule & 22.50 & 2 & 11.25 & 2.74 & 0.0663 & \\
\hline $\begin{array}{l}\text { B-Initial Part } \\
\text { Release }\end{array}$ & 138.33 & 2 & 69.17 & 16.86 & $<0.0001$ & significant \\
\hline C-Buffer & 0.23 & 2 & 0.11 & 0.028 & 0.9727 & \\
\hline$A B$ & 0.99 & 4 & 0.25 & 0.060 & 0.9932 & \\
\hline$A C$ & 2.08 & 4 & 0.52 & 0.13 & 0.9727 & \\
\hline$B C$ & 0.41 & 4 & 0.10 & 0.025 & 0.9988 & \\
\hline Residual & 1029.52 & 251 & 4.10 & & & \\
\hline Lack of Fit & 6.44 & 8 & 0.81 & 0.19 & 0.9919 & not significant \\
\hline Pure Error & 1023.08 & 243 & 4.21 & & & \\
\hline Cor Total & 1194.06 & 269 & & & & \\
\hline
\end{tabular}

Table 10. ANOVA analysis for total run time

The significance of the model is greater than 99\% ( $F$ value 2.23, p-value < $0.0001)$. The above table also shows the p-value of the ANOVA F-test on Machine Efficiency for all experimental factors and interactions. High $F$ values identify the significant factors and interactions. In this case B (Initial Part Release) is the only 
significant model term. The $F$ value of 16.86 indicates its significance. A summary of statistics for the model is presented in Table 8 .

To verify the ANOVA analysis, the plots of normal probability of residuals and residuals versus predicted are presented in Figures 16 and 17. No abnormalities can be seen in the following normal plot, thus the normality assumption is satisfied.

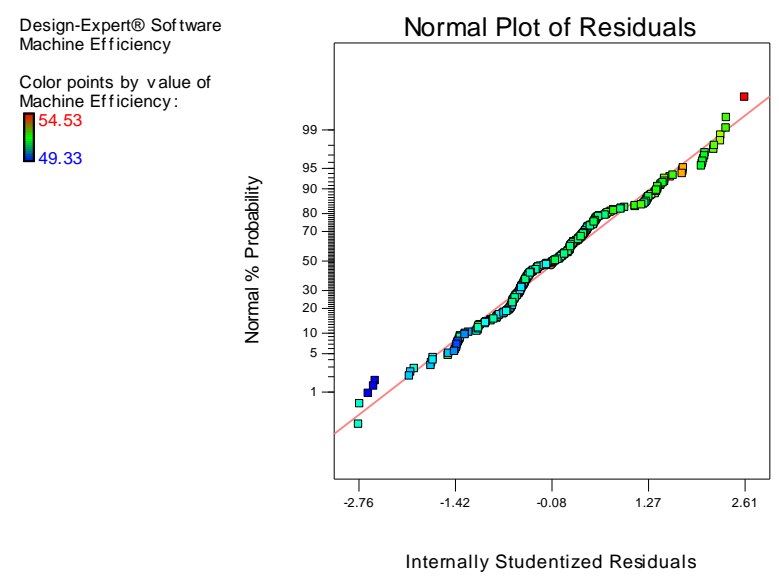

Figure 16. Normal plot of residuals for the ANOVA test on machine efficiency

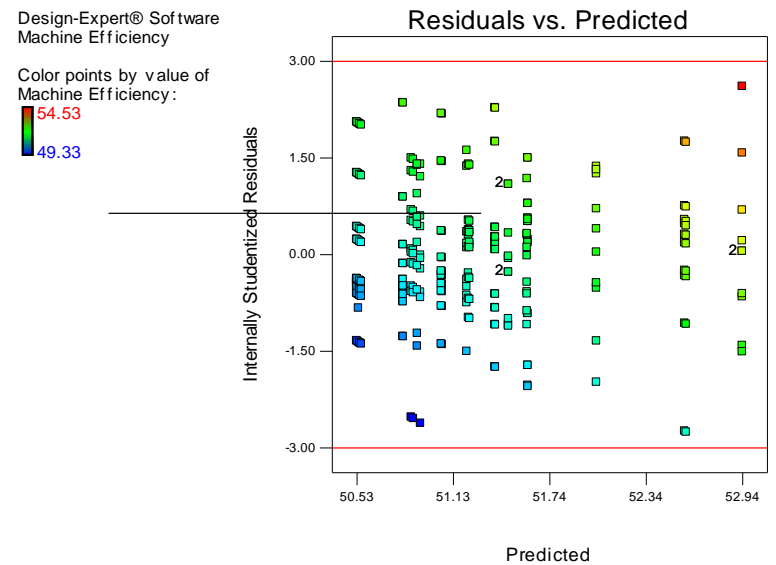

Figure 17. Residuals versus predicted values for machine efficiency

Figures 18 to 20 show the interaction between the initial part release factor and the selected dispatching rule. 


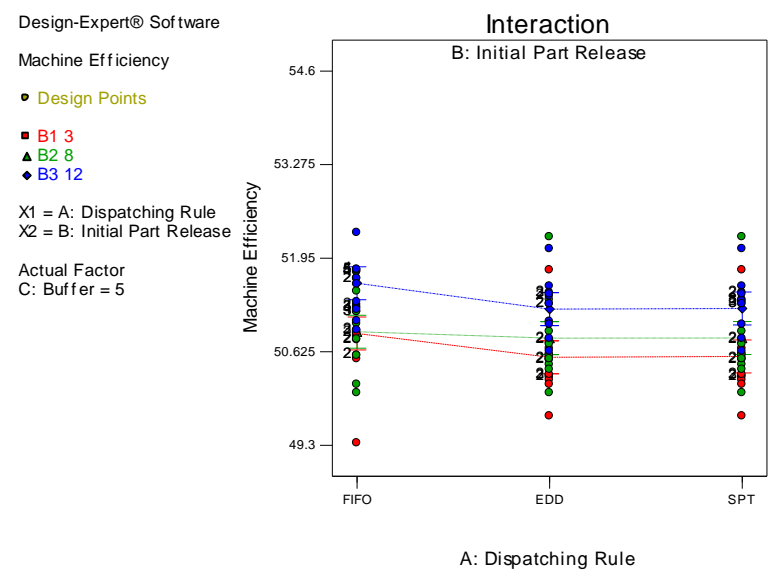

Figure 18. Machine efficiency initial part release $\&$ dispatching rule

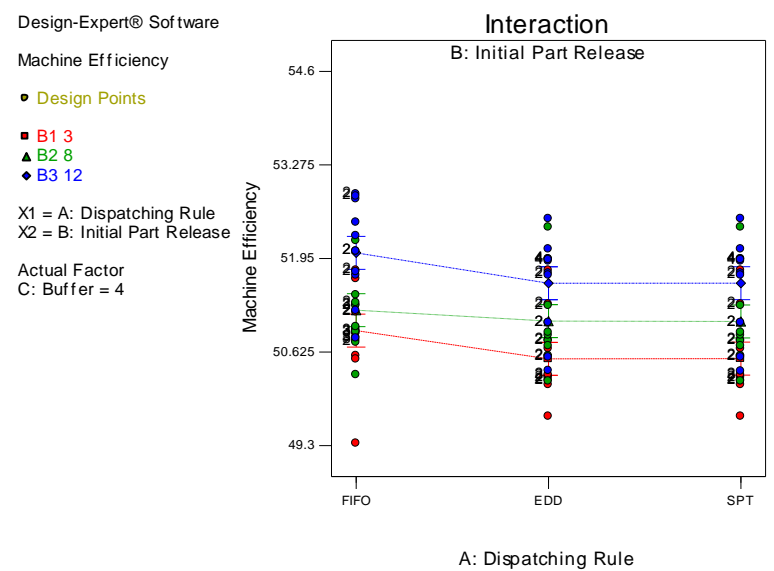

Figure 19. Machine efficiency initial part release $\&$ dispatching rule

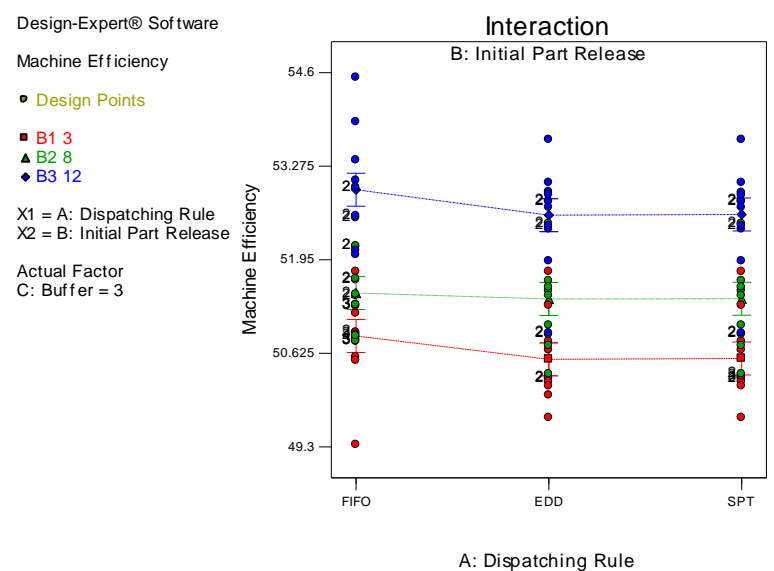

Figure 20. Machine efficiency initial part release $\&$ dispatching rule 
From the graphs, it can be seen that the selected dispatching rule and buffer size do not have a sizeable effect on the total run time. However this is not the case for the initial part release factor. It can be seen that the lower number of initial part release (3) increases the total run time. Yet the highest number of initial part release does not have a significant effect. This can be explained by the fact that at the lowest level of initial part release, there is unused capacity in the CIM system which contributes to the total run time of the production order. Also in the developed model, after the initial release of the parts, each new part enters the system once a final product leaves the system. However for a level of 8 of initial part release and on, there is no extra capacity in the system. Thus increasing the number of initial part release would reduce the total run time.

In summary, according to the aforementioned discussion, the system's total run time performs markedly better when the initial part release is set at eight or higher.

\section{Conclusions and future research}

The objective of this research was to identify the potential effect of a selected number of dispatching rules and two other factors, the number of buffers and initial number of part release, on the performance of the existing CIM systems with different part types where the machines are the major resource constraints.

The following summarizes the findings of this research:

- Selection of the initial part release factor, number of buffers and the dispatching rule has a sizeable effect on the mean flow time of the parts, except for the lower numbers of initial part release. As the number of buffers increases the effect of the dispatching rules on the part Average Flow Time becomes more pronounced at the higher level of initial part release due to formation of a larger queue.

- At a given level of buffer size, there is an optimum number for initial part release which minimizes the part mean flow time. A lower level of initial part release increases the mean flow time.

- The increase in the number of buffers reduces the part Mean Flow Time with more effect on the higher number of the initial part release.

- Moving from the FIFO dispatching rule to the other two dispatching rules, reduces the average flow time of parts. 
- Contrary to the number of initial part release and number of buffers in the system, the selection of the dispatching rule does not have a sizeable effect on machine efficiency.

- A lower initial part release number reduces machine efficiency since the increase in the number of initial parts released would increase the system workload which results in more use of the machines and thus, higher machine efficiency.

- Increases of the number of buffers in the system reduce the effect of the initial number of part release on the machine efficiency, since by having a smaller buffer size; the machines are used more efficiently to finish the job order.

- The selected dispatching rule and buffer size do not have a sizeable effect on the total run time. However this is not the case for the initial part release factor. A lower number of initial parts released increases the total run time. Yet the highest number of initial parts released does not have a significant effect. At the lowest level, there is unused capacity in the CIM system. This fact coupled with the control logic of the CIM system (each new part enters the system once a final product leaves the system) explains this effect.

In summary, the system performs much better considering the Machine Efficiency when the initial part release is maximized and the buffer size is minimum. Furthermore, considering the Average Flow Time, the system performs much better when the selected dispatching rule is either EDD or SPT with buffer size of five and initial part release of eight.

Compared to the literature (Conway, 1965a; Blackstone et al., 1982; Rajendran \& Holthaus, 1999), where it was concluded that the overall best simple dispatching rules among all other simple rules in order of their performance are SPT, EDD and FIFO, our research concludes that the performance of the FIFO is worse than the other two rules however a tangible difference between the SPT and EDD was not observed.

This research is among the few that study the effect of dispatching rules on the performance of the CIM systems using a terminating simulation. This is also significant given the nature of the CIM systems that are mostly used to produce different parts in varying quantities and thus do not produce parts on a continuing basis. This research is among the first to study the combined effect of dispatching 
rules and the buffer size in the CIM systems where the job arrivals are predetermined and depend on the completion of the existing parts in the system. $\mathrm{A}$ description of how buffer size and initial number of parts released is related to the performance of the CIM system for the priority dispatching rule is also provided.

The following are suggested areas for future research. In this research, the proposed work was performed based on a particular example of a FMS system, which reduces the generality of the results. In future work, a general model should be developed to study the current problem. Next, a limited number of factors and levels were considered for the experiment set-up. However the flexibility of the model allows experimenting with additional factors and levels. In the simulation experiments of this research, three scheduling dispatching rules (FIFO, EDD, SPT) were used. In future research, the effect of other dispatching rules on the system performance can be compared. This research also was limited in that only three different part types with similar routing (flow shop) and constant due date were considered. It is possible to introduce additional flexibility into the design of the experiment, including the different part routings (job shop design) and variable due dates and conduct a experiment to see if the results of this experiment will be hold for the those other situations. The simulation model and method used in this paper can be adapted easily to study this. A non-terminating simulation model can also be used as another research direction to compare the results and findings with collected results from the terminating simulation model of this study. Certain assumptions were made during this research. These assumptions can be addressed in further research. One important assumption is that there is no failure in the system. It would be a good idea during future research to consider the effect of machine breakdown. In the simulation experiments of this research it was considered that all the jobs are ready to be dispatched to the system. In future research, it would be interesting to investigate how the system performs when, for example, an exponential distribution is used for the job arrival similar to the majority of the studies in this field. Finally, simulation experiments could also be conducted to study the effect of the dispatching rules on system performance for an assembly shop where two or more parts are used to produce one final product.

\section{References}

Asfahl, C.R. (1992). Robots and manufacturing automation (2nd Ed.). John Wiley \& Sons Inc. 
Blackstone, J.H., Phillips, D.T., \& Hogg, G.L. (1982). State of the art survey of dispatching rules for manufacturing job shop operations. International Journal of Production Research, 20(1), 27-45. http://dx.doi.org/10.1080/00207548208947745

Cagliano, R., \& Spina, G. (2000). Advanced manufacturing technologies and strategically flexible Production. Journal of Operations Management, 18(2), 169190. http://dx.doi.org/10.1016/S0272-6963(99)00022-4

Choi, R.H., \& Malstrom, E.M. (1988). Evaluation of traditional work scheduling rules in a flexible manufacturing system with a physical simulator. Journal of Manufacturing Systems, 7(1), 33-45. http://dx.doi.org/10.1016/0278-6125(88)900313

Chung, C.A. (2004). Simulation modeling handbook: A practical approach. CRC Press.

Conway, R.W. (1965a). Priority dispatching and work-in-process inventory in a job shop. Journal of Industrial Engineering, 16(2), 123-130.

Conway, R.W. (1965b). Priority dispatching and job lateness in a job shop. Journal of Industrial Engineering, 16(4), 228-236.

Drake, G.R., Smith, J.S., \& Peters, B.A. (1995). Simulation as a planning and scheduling tool for flexible manufacturing systems. Winter Simulation Conference, Proceedings of the $27^{\text {th }}$ Conference on Winter Simulation, Arlington, Virginia, USA, 805-812.

Hong, H.C., \& Chou, S.H. (2002). Comparing dispatching rules in an open shop-a simulation study. Proceedings of the 2002 IIE Annual Research Conference, (CDROM, Paper No. 2082).

Holthaus, O., \& Rajendran, C. (1997). New dispatching rules for scheduling in a job shop - an experimental study. International Journal of Advanced Manufacturing Technology, 13(2), 148-153. http://dx.doi.org/10.1007/BF01225761

Jones, A., \& Rabelo, L.C. (1998). Survey of job shop scheduling techniques. Gaithersburg, MD: Technical Report, National Institute of Standards and Technology.

Kelton, W.D., \& Law, A.M. (1984). An analytical evaluation of alternative strategies in steady-state simulation. Operational Research, 32(1), 169-184. http://dx.doi.org/10.1287/opre.32.1.169 
Mohanasundaram, K.M., Natarajan, K., Viswanathkumar, G., Radhakrishnan, P., \& Rajendran, C. (2003). Scheduling rules for dynamic shops that manufacture multi-level jobs. Computer and Industrial Engineering, 44(1), 119-131. http://dx.doi.org/10.1016/S0360-8352(02)00188-2

Montgomery, D.C. (2001). Design and analysis of experiments ( $5^{\text {th }}$ Ed.). John Willey \& Sons Inc.

Montazeri, M., \& Van Wassenhove, L.N. (1990). Analysis of scheduling rules for an FMS. International Journal of Production Research, 28(4), 785-802. http://dx.doi.org/10.1080/00207549008942754

Nagalingam, S.V., \& Lin, G.C.I. (1999). Latest developments in CIM. Robotics and Computer Integrated Manufacturing, 15(6), 423-430. http://dx.doi.org/10.1016/S0736-5845(99)00036-8

OpenCIM (2004). OpenCIM Intro and OpenFMS, computer integrated manufacturing for industrial training applications, software version 4.0, user manual. Catalog No. 100094 Rev. E, Intelitek.

Persi, P., Ukovich, W., Pesenti, R., \& Nicolich, M. (1999). A hierarchic approach to production planning and scheduling of a flexible manufacturing system, Robotics and Computer Integrated Manufacturing, 15(5), 373-385. http://dx.doi.org/10.1016/S0736-5845(99)00034-4

Rajendran, C., \& Holthaus, O. (1999). A comparative study of dispatching rules in dynamic flow shops and job shops. European Journal of Operational Research, 116(1), 156-170. http://dx.doi.org/10.1016/S0377-2217(98)00023-X

Sabuncuoglu, I., \& Lejmi, T. (1999). Scheduling for non regular performance measure under the due window approach. Omega, The International Journal of Management Science, 27(5), 555-568.

Schmeiser, B. (1982). Batch size effects in the analysis of simulation output. Operational Research, 30(3), 556-568. http://dx.doi.org/10.1287/opre.30.3.556

Theodorou, P., \& Florou, G. (2008). Manufacturing strategies and financial performance - the effect of advanced information technology: CAD/CAM systems. Omega, The International Journal of Management Science, 36(1), 107-121. 
Tseng, M.C. (2004). Strategic choice of flexible manufacturing technologies.

International Journal of Production Economics, 91(3), 223-227. http://dx.doi.org/10.1016/j.ijpe.2003.08.010

Yazdi, D. B. (2006). Scheduling of a computer integrated manufacturing system: $A$ simulation study, M.A.Sc. Thesis, Concordia University.

Journal of Industrial Engineering and Management, 2011 (www.jiem.org)

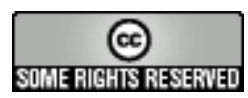

Article's contents are provided on a Attribution-Non Commercial 3.0 Creative commons license. Readers are allowed to copy, distribute and communicate article's contents, provided the author's and Journal of Industrial Engineering and Management's names are included. It must not be used for commercial purposes. To see the complete license contents, please visit http://creativecommons.org/licenses/by-nc/3.0/. 